\title{
Silver-based drug effect on the body of calves with diarrhea
}

\author{
Elena Olentsova ${ }^{1}$, Vladimir Orobets $^{1 . *}$, Olga Sevostyanova ${ }^{1}$, Ivan Kireev $^{1}$, and \\ Alexander Agarkov ${ }^{1}$ \\ ${ }^{1}$ Stavropol State Agrarian University, Zootekhnicheskiy lane, 12. Stavropol, Stavropol reion, Russia, \\ 355017
}

\begin{abstract}
Diarrhea of newborn calves is a globally common disease that causes significant damage to livestock. Economic losses from diarrhea in young cattle are directly related to the cost of long-term treatment of animals and their mortality, as well as the projected decrease in productivity, which negatively affects the efficiency of maintaining the entire herd. Currently, it is an established fact that diarrhea of newborn calves has a polyethological nature, which has an interaction in pathogenesis between the calf's body, enteropathogens and the technology of keeping animals. For the treatment of calves with diarrhea caused by enteropathogenic Escherichia coli, many methods and schemes using various antibiotics have been proposed. The need for fast and efficient treatment for acute diarrhea of calves in many cases leads to an empirical selection of the drug without taking into account the spectrum of its activity. This approach leads to the appearance and rapid spread of antibiotic resistant strains of microorganisms and as a result reduces the effectiveness of veterinary measures. The article presents the results of a study of the effect of a new complex drug containing stabilized silver particles, an immunostimulator and an antioxidant on the body of calves with diarrhea, as an alternative to the antibiotics. Studies were conducted on calves of the first week of life with a diagnosis of diarrhea, with enteropathogenic Escherichia coli confirmation.
\end{abstract}

\section{Introduction}

A necessary condition for the successful development of both dairy and beef cattle breeding is the production and rearing of healthy calves, since the quality of health in the first period of ontogenesis directly affects further development, adaptation to adverse environmental factors and the realization of the genetic potential of productivity. Diarrhea in newborn calves is one of the globally common diseases that cause significant damage to cattle breeding. The economic damage caused by this pathology consists of the cost of medicines for treatment, the death of animals and the loss of potential productivity [1]. In dairy calves, the risk of developing diarrhea varies over time, with a higher total risk of diarrhea occurring during the first two months of life. The maximum risk occurs during the second

\footnotetext{
*Corresponding author: orobets@yandex.ru
} 
week of life [2.3]. However, mortality is higher during the first week after birth [3]. Diarrhea caused by E. coli is the main cause of morbidity and mortality in young animals [4].

In recent years, many methods and schemes using various drugs, both domestic and imported, have been proposed for the prevention and treatment of diarrhea in newborn calves. At the same time, there are very few affordable, cheap, environmentally friendly drugs for effective treatment of this pathology [5]. The widespread use of antibiotics has revealed a number of their negative properties. First, the emergence and rapid spread of antibiotic-resistant strains of microorganisms [4], which encourages investment in the development of new antibacterial drugs and recommendations for the selective use of such drugs. In addition to the appearance of resistant strains, the use of antibiotics acts on the macroorganism negatively affecting the digestive system and secretions, reduces nonspecific resistance and can lead to the development of dysbacteriosis.

According to [5] 88 out of 150 animals with diarrhea tested positive for Escherichia coli. Among them 54 samples had a mixed infection with other bacterial and / or parasitic agents. There are several pathotypes of diarrhea Escherichia coli, including enteropathogenic Escherichia coli (EPEC), Shiga-toxin-producing Escherichia coli (STEC), enterotoxigenic Escherichia coli (ETEC) and necrotoxigenic Escherichia coli (NTEC). Studies have shown that $48(54.5 \%)$ isolates are multidrug-resistant to antibiotics. The percentage of resistance to tetracycline, streptomycin, ampicillin and trimethoprimsulfamethoxazole was 79.5. 67.0. 54.5 and 43.0. respectively. Ceftazidim (14.8\%), amoxicillin-clavulanic acid (13.6\%) and aztreonam (11.3\%) showed the lowest resistance, and none of the isolates were resistant to imipenem [6].

The prevalence of E. coli-related diarrhea in calves was $85.04 \%$, where $35.01 \%$ were caused by enterotoxigenic strains Escherichia coli. The highest antimicrobial resistance was observed for tetracycline (63.21\%) and ampicillin $(48.11 \%)$, while the most active antibiotics were chloramphenicol, gentamicin (96.33\%) and imipenem (99.06\%). Multidrug resistance was detected in $69.81 \%$ of isolates by enterotoxigenic Escherichia coli strains from diarrheal calves. The higher prevalence of genes eaeA and hlyA, carring isolates of enterotoxigenic strains Escherichia coli, may pose a serious zoonotic threat, and the increased prevalence of multidrug resistance in E. coli may require strict selection of an appropriate antimicrobial agent in the treatment of diarrhea in calves [7.8].

Based on the results of an antibioticogram of the selected strains of seven serogroups, it was found that most $E$. coli serotypes show multidrug resistance to four antimicrobial agents; neomycin, gentamicin, streptomycin and amikacin. Enrofloxacin, florfenicol, amoxicillin-clavulanic acid and ampicillin-sulbactam are the most effective antimicrobials against isolated strains STEC and ETEC E. coli [9].

In the study a total of $379 \mathrm{E}$. coli strains were isolated from calves with diarrhea. All $E$. coli isolates were tested for their sensitivity to 15 antimicrobial agents using the KirbyBauer method. Isolates showed the highest levels of resistance to ampicillin $(64.9 \%)$, as well as streptomycin $(59.4 \%)$, tetracycline $(53.8 \%)$, sulfamethoxazole / trimethoprim (50.9\%), chloramphenicol (45.6\%), kanamycin (44.1\%) and enrofloxacin (42.0\%). E. coli isolates showed lower resistance to ceftazidime (15.0\%) and polymyxin (12.6\%) [10].

Shiga-toxigenic E. coli O157: H7 - this is a type of Shiga toxin E. coli, which can cause diseases in humans and animals. In studies [11], the sensitivity test of isolates showed that all isolated cultures are highly resistant to erythromycin (100\%) and cephalotin $(96.87 \%)$. Less resistance to tetracycline, trimethoprim-sulfamethoxazole and chloramphenicol at different percentages $(31.25 \%),(12.5 \%)$ and $(9.37 \%)$, respectively, and all isolates showed high sensitivity to gentamicin (100\%), enrofloxacin (100\%). The emergence of multidrugresistant microbes is facilitated by drug and / or antibiotic resistance, which is acquired by microbes for their survival and reproduction in inconvenient conditions. Multidrug-resistant 
bacterial infections lead to a significant increase in mortality, morbidity, and the cost of long-term treatment. Therefore, the development, modification, or search for antimicrobial compounds with bactericidal potential against multidrug-resistant bacteria is a priority area of research [12].

The above-mentioned numerous evidence of antibiotic resistance and the resulting decrease in treatment effectiveness stimulate the search for alternative therapies. Plant metabolites can be an alternative method of control. In the study [13], Cerrado plant extracts were selected and characterized, demonstrating inhibitory effects against Escherichia coli and Staphylococcus spp. from cattle.

Bacterial probiotic drugs play an important role in restoring normal intestinal microbiocenosis [14]. It was found that to stimulate the recovery of liver function in toxic dyspepsia in newborn calves, it is advisable to introduce an additive containing phospholipids [15].

Many studies conducted by both Russian and foreign scientists have shown that silver nanoparticles have unique physical, chemical, and biomedical properties, which led to their use in human and veterinary medicine, and sanitation. The main property is high bactericidal activity due to bacterial inactivation and growth inhibition [16.17], spreading to more than 600 species of bacteria, viruses and fungi [18].

An in vitro study of the antibacterial activity of silver nanoparticles obtained by the modified Tollens process revealed high antibacterial activity even at very low concentrations of several units $\mathrm{mg} / \mathrm{l}$. These concentrations are comparable to those of ionic silver, showing the same antibacterial effect. However, such low concentrations of silver nanoparticles do not show acute cytotoxicity to mammalian cells - this occurs at silver concentrations above $60 \mathrm{mg} / 1$, while the cytotoxic level of ionic silver is much lower (approximately $1 \mathrm{mg} / 1$ ) [19].

The inhibitory effect of silver is probably the sum of the various mechanisms of action. A number of studies suggest that silver ions react with SH-groups of microbial proteins [20]. The presence of silver and sulfur elements in electron-dense granules and cytoplasm detected by x-ray microanalysis suggests an antibacterial mechanism of silver: DNA loses its ability to replicate, and the protein becomes inactivated after $\mathrm{Ag}(+)$ treatment. more minor morphological changes in $S$. aureus compared to $E$. coli recommended the $S$. aureus protection system from the inhibitory action of $\mathrm{Ag}(+)$ ions[22].

The study [23] was aimed at evaluating the antibacterial effectiveness of biosynthesis of silver nanoparticles using natural honey against bacteria isolated from calves with diarrhea. Most of the bacteria isolated from calves have been identified as E. coli, Salmonella spp, and $S$. aureus. All of these bacteria were sensitive to ciprofloxacin, gentamicin, and chloramphenicol, but were resistant to penicillin, ampicillin, and amoxicillin. The antibacterial effects of silver nanoparticles on multidrug-resistant bacteria had similar or superior effects of ciprofloxacin and chloramphenicol.

Silver nanoparticles treated with a phosphate buffer, as well as carbon-coated nanoparticles, were toxic to all bacterial strains, with the exception of Ag-resistant E. coli, at concentrations between 64.0-1024.0 micrograms / ml. Ag-resistant E. coli died only when using silver nanoparticles not treated with a phosphate buffer or its supernatant, both of which contained formaldehyde [24]. Thus, it becomes obvious that the search for medicines that contain silver for the treatment of diseases in calves with an established infectious pathogen is promising.

The aim of the research was to study the therapeutic properties of a new complex drug based on silver and its effect on the body of calves with diarrhea. 


\section{Materials and Methods}

A study of the therapeutic effectiveness of a drug based on stabilized silver particles, in combination with an immunostimulator and an antioxidant, was conducted on 24 calves of the first week of life with a diagnosis of diarrhea. The calves were divided into two groups based on the principle of analogues. During the study of morphological and biochemical parameters of blood, the data obtained were compared with those of clinically healthy calves $(\mathrm{n}=12)$.

Diarrhea in newborn calves was diagnosed comprehensively taking into account epizootic data, clinical animal studies, morphological and biochemical blood tests, and bacteriological studies of samples from sick calves with the release of enteropathogenic $E$. coli.

Calves were assigned a half-starved diet, skipping the next two drinks of colostrum and replacing them with saline solution. Next feeding portions of milk were reduced by $40 \%$, filling the volume with saline solution 15 minutes before feeding. The drug based on stabilized silver particles was administered to animals of the first group at a dose of 1.5 $\mathrm{ml} / \mathrm{kg}$ of body weight for three to five days. In the treatment of calves from the second group, an antibiotic from the group of third-generation fluoroquinolones was used in accordance with the instructions.

The animals were monitored, noting their clinical condition and clinical course. The criteria for evaluating the effectiveness of therapy for calves with diarrhea were clinical, biochemical, and morphological blood parameters, the duration of treatment, and the time of recovery. Morphological studies were carried out using the veterinary hematological analyzer Mindray BC-2800Vet, biochemical - using an automatic photometric Analyzer CHEM WELL 2910V (C) (Combi).

\section{Results}

According to the data presented in table 1. it can be concluded that the use of a silver-based drug provides a high therapeutic effect and a short treatment period, in comparison with an antibiotic used in the farm for the treatment of calves with diarrhea.

Table 1. Results of the study of comparative medical effectiveness of therapy in calves with diarrhea.

\begin{tabular}{|l|c|c|}
\hline \multicolumn{1}{|c|}{ Indicator } & Group №1 & Group №2 \\
\hline Number of animals in the group & 12 & 12 \\
\hline Course of treatment, days & $4.91 \pm 0.7$ & $5.91 \pm 0.8$ \\
\hline Calves recovered (\%) & $12(100)$ & $11(91.6)$ \\
\hline Calves died (\%) & & $1(8.3)$ \\
\hline Average daily weight gain, g & $567.2 \pm 12.6^{*}$ & $522.4 \pm 18.2$ \\
\hline Recovery time, days & $6.2 \pm 0.7$ & $7.4 \pm 0.5$ \\
\hline Note: ${ }^{*}$ - $<0.05$ & &
\end{tabular}

The therapeutic effectiveness of treatment of calves using a silver-based drug was $100 \%$, and in the group where the "standard" antibiotic was used $-91.6 \%$. At the same time, the course of treatment in the first group was 4.91 days, in the second group - 5.91 days. As a result of the therapy, the improvement of the general condition and the disappearance of clinical and diagnostic manifestations of the disease were registered in the 
first group after 6.2 days, the second - after 7.4 days. The high effectiveness of the studied drug is also confirmed by the indicators of animal productivity. During the 30-day followup period, the average daily growth of animals in the first group was $7.9 \%$ higher than that of calves in the second group.

The results of the conducted morphological researches of blood of sick calves showed that before treatment due to dehydration we found a significant increase in the number of red blood cells in comparison with the control group of animals (table 2). The use of the studied drugs contributed to the normalization of the red blood cells number in calves of the first and second groups. Before treatment, calves with diarrhea showed a decrease in the hemoglobin content in the blood to $94.8 \mathrm{~g} / \mathrm{l}$. Determination of this indicator 15 days after treatment indicated an increase in the level of hemoglobin in animals of the first group by $17.8 \%$, in calves of the second group-by $10.7 \%$.

Table 2. Hematological indicators of calves $(n=10)$.

\begin{tabular}{|c|c|c|c|}
\hline Indicator & Group & Before treatment & 15 days after treatment \\
\hline \multirow{3}{*}{ RBC, $10^{12} / 1$} & 1 & $8.42 \pm 0.38^{*}$ & $7.71 \pm 0.25$ \\
\cline { 2 - 4 } & 2 & $8.38 \pm 0.25^{*}$ & $8.03 \pm 0.38$ \\
\cline { 2 - 4 } & Control & $7.23 \pm 0.32$ & $7.45 \pm 0.38$ \\
\hline \multirow{3}{*}{ Hemoglobin, g/1 } & 1 & $95.20 \pm 3.22^{*}$ & $112.20 \pm 3.28$ \\
\cline { 2 - 4 } & 2 & $94.51 \pm 2.35^{*}$ & $104.62 \pm 2.31$ \\
\cline { 2 - 4 } & Control & $113.12 \pm 3.02$ & $113.56 \pm 3.02$ \\
\hline \multirow{3}{*}{ WBC, $10^{9} / 1$} & 1 & $10.68 \pm 0.85^{*}$ & $7.61 \pm 0.87$ \\
\cline { 2 - 4 } & 2 & $10.53 \pm 0.28^{*}$ & $8.33 \pm 0.58$ \\
\cline { 2 - 4 } & Control & $7.46 \pm 0.25$ & $7.54 \pm 0.24$ \\
\hline
\end{tabular}

The study of the content of white blood cells in the peripheral blood of calves allowed us to state leukocytosis in sick animals, confirming the development of an inflammatory process in the gastrointestinal tract. The level of white blood cells in the blood of calves of the first and second groups was significantly higher than the same indicator in clinically healthy calves by 43.2 and $41.1 \%$, respectively. 15 days after treatment, the number of white blood cells in the first group decreased to $7.61 \cdot 10^{9} / 1$, in the second -to $8.33 \cdot 10^{9} / 1$, respectively.

The leukogram of sick animals is characterized by changes in cellular composition against the background of functional digestive disorders, accumulation of a significant amount of ammonia and residual nitrogen, which cause the development of alimentary toxicosis. In addition, toxicosis of the body of calves occurs due to the absorption of toxins that are released in large quantities by enteropathogenic bacteria (table 3 ).

The number of basophils in all calves was within the limits of the physiological norm. However, before treatment, the number of basophils in calves with diarrhea was on average significantly higher in comparison with healthy animals by 2.5-3 times. The number of eosinophils in the blood all the days of the study was within the refractive values. Before the treatment, an increase in immature and rod-shaped neutrophils was observed in sick calves. The significant excess of the number of immature neutrophils in sick animals was 2.6-3 times, and the number of rod-shaped neutrophils was 1.4 times. 
Table 3. Leukocyte formula in calves, $\%(n=10)$.

\begin{tabular}{|c|c|c|c|}
\hline Indicator & Group & Before treatment & 15 days after treatment \\
\hline \multirow{3}{*}{ Basophils 0-2 } & 1 & $0.5 \pm 0.02^{*}$ & $0.3 \pm 0.05^{*}$ \\
\cline { 2 - 4 } & 2 & $0.6 \pm 0.01^{*}$ & $0.4 \pm 0.04^{*}$ \\
\cline { 2 - 4 } & Control & $0.2 \pm 0.04$ & $0.3 \pm 0.08$ \\
\hline \multirow{3}{*}{ Eosinophils 5-8 } & 1 & $7.1 \pm 0.42$ & $6.8 \pm 0.24$ \\
\cline { 2 - 4 } & 2 & $6.9 \pm 0.35$ & $6.6 \pm 0.26$ \\
\cline { 2 - 4 } & Control & $6.5 \pm 0.38$ & $6.4 \pm 0.36$ \\
\hline \multirow{3}{*}{ immature 0-1 } & 1 & $0.8 \pm 0.03^{*}$ & $0.5 \pm 0.07$ \\
\cline { 2 - 4 } & 2 & $0.9 \pm 0.02^{*}$ & $0.5 \pm 0.06$ \\
\cline { 2 - 4 } rod-nuclear 2-5 & Control & $0.3 \pm 0.06$ & $0.2 \pm 0.04$ \\
\cline { 2 - 4 } & 1 & $5.2 \pm 0.38^{*}$ & $3.8 \pm 0.42$ \\
\cline { 2 - 4 } & Control & $5.1 \pm 0.72^{*}$ & $3.7 \pm 0.68$ \\
\hline \multirow{3}{*}{ segmentonuclear } & 1 & $31.7 \pm 0.62$ & $3.6 \pm 0.52$ \\
\cline { 2 - 4 } 20-35 & 2 & $31.4 \pm 2.31$ & $33.3 \pm 2.33$ \\
\cline { 2 - 4 } & Control & $31.7 \pm 2.35$ & $34.6 \pm 2.65$ \\
\hline \multirow{3}{*}{$\begin{array}{c}|c| \\
\text { Lymphocytes }\end{array}$} & 1 & $46.2 \pm 3.42^{*}$ & $50.4 \pm 2.17$ \\
\cline { 2 - 4 } & 2 & $45.8 \pm 3.25^{*}$ & $49.5 \pm 4.12$ \\
\cline { 2 - 4 } & Control & $53.1 \pm 4.46$ & $52.7 \pm 3.52$ \\
\hline \multirow{3}{*}{\begin{tabular}{c} 
Monocytes 2-7 \\
\cline { 2 - 4 }
\end{tabular}} & 2 & $9.2 \pm 1.24^{*}$ & $4.9 \pm 1.18$ \\
\cline { 2 - 4 } & Control & $4.3 \pm 1.32^{*}$ & $5.2 \pm 1.14$ \\
\hline
\end{tabular}

Note: $*-p<0.05$ the difference is statistically significant between given and the control group.

An increase in the level of neutrophils in this case can characterize the development of acute bacterial infection, when the production of neutrophils increases significantly, immature forms of neutrophils enter the bloodstream, resulting in an increase in the number of immature and rod-shaped neutrophils. This shift of the leukocyte formula to the left indicates the activity of the bone marrow response to infection. The number of segmentonuclear neutrophils in the blood of clinically healthy and sick calves was normal throughout the study period. In percentage terms, a significant decrease in the number of lymphocytes in calves with diarrhea by $13-13.7 \%$ and an excess of the reference values for the percentage of monocytes in sick animals in comparison with control animals by 31.4$32.8 \%$ was registered within the limits of physiological values. A decrease in the level of lymphocytes and an increase in the percentage of monocytes characterizes the development of acute bacterial infection in calves with diarrhea.

In the biochemical profile of sick calves, a significant decrease in total protein, reserve alkalinity and glucose, and an increase in the concentration of total calcium and inorganic phosphorus was found in comparison with clinically healthy animals. The decrease in the total protein content in sick animals is probably associated with a shift in the acid-base balance to the acidic side, the formation of under-oxidized metabolic products that violate the metabolic function of the liver, which is manifested in a violation of protein synthesis, including plasma proteins. Differences in indicators between sick and healthy calves were noted not only in the content of total protein, but in the quantitative composition of its fractions. Violation of protein synthesis in the liver of sick calves leads to a violation of the normal ratio of protein fractions in the blood serum. Thus, before treatment, the albumin content was significantly lower than the values in control animals by $30.8 \%$, and globulins, respectively, were higher than $\alpha$ by $21.2 \%, \beta$ - by $5.1 \%$, and $\gamma$-by $9.4 \%$ (table 4 ). 
Table 4. Biochemical parameters of blood serum in calves $(n=10)$.

\begin{tabular}{|c|c|c|c|}
\hline Indicator & Group & Before treatment & 15 days after treatment \\
\hline \multirow[t]{3}{*}{ Crude protein, $g / 1$} & 1 & $62.40 \pm 2.08^{*}$ & $71.00 \pm 1.10$ \\
\hline & 2 & $63.50 \pm 2.12 *$ & $69.90 \pm 1.22$ \\
\hline & Control & $70.20 \pm 1.64$ & $71.10 \pm 1.64$ \\
\hline \multirow[t]{3}{*}{ Albumins $g / 1$} & 1 & $25.30 \pm 2.27^{*}$ & $37.40 \pm 0.78$ \\
\hline & 2 & $26.20 \pm 2.00^{*}$ & $36.90 \pm 0.86$ \\
\hline & Control & $37.20 \pm 1.34$ & $37.50 \pm 2.15$ \\
\hline \multirow[t]{3}{*}{$\alpha$-globulins, g/l } & 1 & $12.50 \pm 1.42 *$ & $10.50 \pm 0.24$ \\
\hline & 2 & $12.70 \pm 1.33^{*}$ & $10.30 \pm 0.35$ \\
\hline & Control & $10.40 \pm 0.65$ & $10.60 \pm 0.45$ \\
\hline \multirow[t]{3}{*}{$\beta$ - globulins, $g / 1$} & 1 & $11.50 \pm 0.75$ & $10.70 \pm 0.67$ \\
\hline & 2 & $11.00 \pm 0.88$ & $10.50 \pm 0.65$ \\
\hline & Control & $10.70 \pm 1.17$ & $10.60 \pm 1.25$ \\
\hline \multirow[t]{3}{*}{$\gamma$-globulins, $\mathrm{g} / \mathrm{l}$} & 1 & $13.10 \pm 0.56^{*}$ & $12.40 \pm 0.53$ \\
\hline & 2 & $13.60 \pm 0.79^{*}$ & $12.20 \pm 1.03$ \\
\hline & Control & $12.20 \pm 0.25$ & $12.00 \pm 0.43$ \\
\hline \multirow{3}{*}{$\begin{array}{l}\text { Reserve } \\
\text { alkalinity, \% } \\
\mathrm{CO}_{2}\end{array}$} & 1 & $36.50 \pm 3.10^{*}$ & $50.40 \pm 2.20$ \\
\hline & 2 & $34.30 \pm 3.50^{*}$ & $47.80 \pm 2.10$ \\
\hline & Control & $51.00 \pm 2.60$ & $50.80 \pm 2.15$ \\
\hline \multirow[t]{3}{*}{ Glucose, $\mathrm{mmol} / \mathrm{l}$} & 1 & $2.72 \pm 0.34 *$ & $5.06 \pm 0.18$ \\
\hline & 2 & $3.02 \pm 0.42 *$ & $5.00 \pm 0.23$ \\
\hline & Control & $5.14 \pm 0.26$ & $5.08 \pm 0.32$ \\
\hline \multirow{3}{*}{$\begin{array}{l}\text { Total calcium, } \\
\mathrm{mmol} / \mathrm{l}\end{array}$} & 1 & $4.70 \pm 0.36^{*}$ & $3.05 \pm 0.19$ \\
\hline & 2 & $4.50 \pm 0.27 *$ & $3.19 \pm 0.34$ \\
\hline & Control & $3.12 \pm 0.22$ & $3.09 \pm 0.24$ \\
\hline \multirow{3}{*}{$\begin{array}{l}\text { Inorganic } \\
\text { phosphorus, } \\
\text { mmol / 1 }\end{array}$} & 1 & $2.21 \pm 0.27^{*}$ & $1.21 \pm 0.18$ \\
\hline & 2 & $2.30 \pm 0.23^{*}$ & $1.34 \pm 0.16$ \\
\hline & Control & $1.25 \pm 0.25$ & $1.12 \pm 0.20$ \\
\hline
\end{tabular}

Note: ${ }^{*}-\mathrm{p}<0.05$ the difference is statistically significant between given and the control group

Reserve alkalinity in sick calves before treatment was reduced by $44.1 \%$, which may also indicate a shift in the alkaline balance towards acidosis against the background of changes in the $\mathrm{H}^{2} \mathrm{O}$ balance and electrolytes. The glucose level in animals of the first and second groups before treatment was at the level of $2.72 \pm 0.34$ and $3.02 \pm 0.42 \mathrm{mmol} / \mathrm{l}$ with the value of this indicator in the control group of $5.14 \pm 0.26 \mathrm{mmol} / 1$, which reflects a violation of gluconeogenesis in the sick animals body. The development of diarrhea leads to significant violations of water-salt metabolism in the body of animals. The amount of total calcium in the blood serum of calves with diarrhea before treatment in the first and second groups was significantly higher than the control indicators and was 4.7 and 4.5 $\mathrm{mmol} / \mathrm{l}$, decreasing to $3.19 \pm 0.34$ and $3.09 \pm 0.24 \mathrm{mmol} / \mathrm{l}$, respectively, 15 days after treatment. The amount of inorganic phosphorus also exceeded the norm in sick calves by almost 1.8 times, reducing to unreliable differences with the values of the control group by the $15^{\text {th }}$ day after treatment.

\section{Discussion}

Maintaining the characteristics of the herd at the proper level is achieved by preventing and controlling diarrhoea in newborns. Evaluation of methods of keeping herds and farms, correct diagnostics of pathogens and analysis of the quality and consumption of colostrum are the most important steps to solve this problem, which causes great damage to dairy farming. Choosing the right prevention/treatment program is also critical to success [1]. When detecting specific pathogens, the use of antimicrobial drugs is a mandatory 
component of the complex of measures in the treatment of calf diarrhea. The effectiveness of antibiotics in many cases is reduced due to the increasing resistance of microorganisms to drugs resulting from the selective effect of antimicrobial drugs on various structures of the microbial cell.

As an alternative, the effectiveness of a drug based on stabilized silver particles in combination with an immunostimulator and an antioxidant was studied in complex treatment regimens for calf diarrhea.

The use of the drug at a dose of $1.5 \mathrm{ml} / \mathrm{kg}$ of body weight for three to five days provides a high therapeutic effect and can be used to treat gastrointestinal diseases of calves complicated by infection in the first days of life. Application the drug provides normalization of morphological and biochemical parameters of blood of sick calves.

\section{Conclusions}

The use of a drug based on stabilized silver particles, in combination with an immunostimulator and an antioxidant, reduces the recovery time of calves with diarrhea, increases the safety of animals. The pronounced clinical effect of the drug causes relatively higher productivity indicators of calves by average daily weight gain.

\section{References}

1. Diarrhea of newborn calves. Practical guide. Intervet International B.V. 126 (2011) ISBN: 978-90-801886-0-0.

2. A. M. K Virtala, G.D. Mechor, Y.T. Gröhn, N.E. Hollins Morbidity from nonrespiratory diseases and mortality in dairy heifers during the first three months of life. $J$ Am Vet Med Assoc; 208 (12), 2043 - 6. (1996)

3. S.J. Wells, L.P. Garber, G.W. Hill Health status of preweaned dairy heifers in the United States Prev Vet Med; 29, 185 - 99 (1996)

4. I. Salhi, S. Bessalah, Snoun, D, Khorchani, T, Hammadi, M. Construction of a nanobodies phage display library from an escherichia coli immunized dromedary. Iranian, Journal of Biotechnology, 18(1), e2247, 16-23 (2020)

5. A.A Aliev, M.G Khalipaev, B.M Gadzhiev, Azizov, I.M, Dzhamaludinov, N.M. Treatment of dyspepsy in calves with an innovative drug - iodpropionix. EurAsian Journal of BioSciences, 14(1), 173-176 (2020)

6. N.M Sobhy, S.G.A Yousef, H.A Aboubakr, Virulence factors and antibiograms of Escherichia coli isolated from diarrheic calves of Egyptian cattle and water buffaloes. PLoS ONE. 15(5), e0232890 (2020)

7. M Srivani, Srinivasa Rao, T. Prevalence and antimicrobial resistance pattern of Shiga toxigenic Escherichia coli in diarrheic buffalo calves. Veterinary World. 10(7), 774778 (2017)

8. W.S Mousa, Abo Shama, U.H. Prevalence, antimicrobial resistance and substantial virulence-associated genes of Escherichia coli isolated from colibacillosis in neonatal calves in Egypt, Journal of Microbiology, Biotechnology and Food Sciences. 9(6), c. 1145-1150 (2020)

9. A.M Algammal, El-Kholy, A.W, Riad, E.M, Hozzein, W.N, Ghobashy, M.O.I. Genes encoding the virulence and the antimicrobial resistance in enterotoxigenic and shigatoxigenic E. coli isolated from diarrheic calves. Toxins, 12(6), 383 (2020) 
10. X. Zhang, J. Li, L. Zhang, Phylogenetic grouping and antimicrobial resistance profiles of escherichia coli isolated from calves in Xinjiang, China. Kafkas Universitesi Veteriner Fakultesi Dergisi. 26(3) (2020)

11. M.S Hasan, M.A Hussein, A.A. Yousif, Confirmatory detection of Escherichia coli o157:H7 in diarrhoeic and non-diarrhoeic calves by using real time PCR with studying the antimicrobial susceptibility of these bacteria. Research, Journal of Pharmacy and Technology, 12(1), 245-250 (2019)

12. M.K Rai, S.D Deshmukh, Silver nanoparticles: The powerful nanoweapon against multidrug-resistant bacteria, Journal of Applied Microbiology. 112(5), c. 841-852 (2012)

13. de O. Ribeiro, I.C, Mariano, E.G.A, Careli, R.T, De Souza, M.R, Duarte, E.R. BMC Veterinary Research. Plants of the Cerrado with antimicrobial effects against Staphylococcus spp. and Escherichia coli from cattle. 14(1), 32 (2018)

14. A.A Elenshleger, A.I Lelak, G.A Nozdrin, A.B. Trebukhov, The effect of probiotic Vetom 2 on the microbial intestinal landscape in calves after antibiotic therapyThe effect of probiotic Vetom 2 on the microbial intestinal flora in calves after antibiotic therapy, IOP Conference Series: Earth and Environmental Science, 341(1),012150 (2019).

15. V.A Gryshchenko, T.M Chernyshenko, O.V Gornitska, Platonova, T.M. Evaluation of the functional state of liver and the efficiency of therapy for enteropathy of calves. Fiziolohichnyi zhurnal. 62(6), 102-109 (2016)

16. Dror-Ehre, A, Mamane, H, Belenkova, T, Markovich, G, Adin, A. Silver nanoparticleE. coli colloidal interaction in water and effect on E. coli survival, Journal of Colloid and Interface Science. 339(2), 521-526 (2009)

17. M.K Rai, S.D Deshmukh, A.P Ingle, Silver nanoparticles: The powerful nanoweapon against multidrug-resistant bacteria, Journal of Applied Microbiology, 112(5), 841-852 (2012)

18. L. N. Podlegaeva, D. M. Russakov, S. A. Sozinov Examination of silver nanoparticles properties obtained by reduction from solutions and thermal deposition in vacuum Bulletin. №2. 95-96 (2009)

19. L Kvitek, A Panacek, Antibacterial activity and toxicity of silver - Nanosilver versus ionic silver, Journal of Physics: Conference Series, 304(1), 012029 (2011)

20. S. Y. Liau, D. C. Read Interaction of silver nitrate with readily identifiable groups: relationship to the antibacterial action of silver ions, Lett Appl Microbiol. 25(4):279-83 (1997)

21. J. R Morones, J. L Elechiguerra bactericidal effect of silver nanoparticles. Nanotechnology, 16(10):2346-53 (2005)

22. Q. L. Feng, J. Wu, G. Q. Chen, F. Z. Cui A mechanistic study of the antibacterial effect of silver ions on Escherichia coli and Staphylococcus aureus. J Biomed Mater Res, 52(4):662-8 (2000)

23. G.S.G Zeedan, A.M Abdalhamed, E.S Ibrahim, Antibacterial efficacy of green silver nanoparticles against bacteria isolated from calf diarrhea, Asian Journal of Epidemiology, 11(2), 65-73 (2018)

24. M.E Samberg, P.E Orndorff,. Antibacterial efficacy of silver nanoparticles of different sizes, surface conditions and synthesis methods. Nanotoxicology. 5(2), 244-253 (2011) 